\title{
Concentration-dependent optical properties of erbium doped zirconia nanocrystals
}

\author{
Timur Sh. Atabaev ${ }^{1, ~ *, ~ M a k i o ~ K u r i s u ~}{ }^{2}$, Kensuke Konishi ${ }^{2}$, Nguyen Hoa Hong ${ }^{1, \text { * }}$ \\ ${ }^{1}$ Department of Physics and Astronomy, Seoul National University, Seoul, Korea \\ ${ }^{2}$ Department of Physics, Ehime University, Matsuyama, Japan
}

Email address:

atabaev@snu.ac.kr (T. S. Atabaev),nguyenhong@snu.ac.kr (N. H. Hong)

\section{To cite this article:}

Timur Sh. Atabaev, Makio Kurisu, Kensuke Konishi, Nguyen Hoa Hong. Concentration-Dependent Optical Properties of Erbium Doped Zirconia Nanocrystals. American Journal of Nanoscience and Nanotechnology. Vol. 2, No. 1, 2014, pp. 13-16.

doi: 10.11648/j.nano.20140201.13

\begin{abstract}
This study has investigated the effect of dopant concentration on the luminescent emission of $\mathrm{Er}^{3+}$ in $\mathrm{ZrO}_{2}$ nanocrystals. The structure and morphology of the resulting nanocrystals were characterized by X-ray diffraction and field emission scanning electron microscope. The room-temperature optical properties of synthesized nanocrystals were studied by photoluminescence spectroscopy. The dependence of the luminescence emission on the doping concentration was examined to determine the optimum $\mathrm{Er}^{3+}$ concentration in the samples. Strong luminescence quenching was also observed in samples with high $\mathrm{Er}^{3+}$ concentrations in the $\mathrm{ZrO}_{2}$ host lattice. The luminescent erbium doped zirconia nanocrystals are promising for applications such as fluorescent biomarkers, optical display systems, lamps, etc.
\end{abstract}

Keywords: Zirconia, Er $^{3+}$ Doped, Luminescence, Nanocrystals

\section{Introduction}

Rare-earth (RE) ions have played an important role in the development of optical materials during the past few decades. The emission wavelength of RE-doped optical materials is independent of particle size and depends only on dopant type, leading to lower synthesis cost. Among them, erbium $\mathrm{Er}^{3+}$ doped luminescent nanomaterials are particularly attractive from both practical and fundamental viewpoints owing to their unique optical properties arising from intra $4 f$ transitions, which gives a strong and sharp visible green emission. These characteristics make them a good alternative to quantum dots (QD) for the development of optoelectronic devices, biomarkers, electroluminescent devices, etc.

Low lattice phonon energy can greatly reduce the non-radiative decay rate of multiphonon relaxations, and can thus effectively enhance the luminescence emission [1]. Furthermore, the efficiency of RE-doped phosphors for frequency conversion are often influenced by changing the dopant concentration [2]. Among the oxide hosts, the zirconia $\mathrm{ZrO}_{2}$ matrix seems to be an ideal host material, because the $\mathrm{ZrO}_{2}$ crystal has a low phonon energy (470 $\mathrm{cm}^{-1}$ ), which opens up the possibility to obtain highly efficient luminescence of active ions incorporated into the matrix. Moreover, the $\mathrm{ZrO}_{2}$ matrix is chemically, photo-thermally, and photo-chemically stable and has a broad optical transparence from the visible to the NIR [3].

From the fundamental point of view, the physical understanding of the frequency conversion process of RE-doped nanocrystals and how it changes with size, crystal phase and concentration is very important. Therefore, our main objective is to study the frequency conversion of $\mathrm{Er}^{3+}$ in $\mathrm{ZrO}_{2}$ matrix and the effects of varying dopant concentration and excitation wavelength. Herein, erbium doped zirconia nanocrystals were prepared using the large-scale urea homogeneous precipitation method. The luminescent properties of erbium doped zirconia nanocrystals were investigated systematically, and found to be strongly dependent on the $\mathrm{Er}^{3+}$-concentration, that can be easily controlled during the fabrication.

\section{Methodology}

Analytical grade zirconium (IV) chloride $\mathrm{ZrCl}_{4}$ (99.5\%), erbium (III) nitrate pentahydrate $\mathrm{Er}\left(\mathrm{NO}_{3}\right)_{3} \cdot 5 \mathrm{H}_{2} \mathrm{O}(99.9 \%)$, and urea $\mathrm{NH}_{2} \mathrm{CONH}_{2}(99.0 \%-100.5 \%)$ were purchased from Sigma-Aldrich Corporation (MO, USA) and were 
used without further purification.

\subsection{Synthesis of $\mathrm{Er}^{3+}$ Doped Zro $\mathrm{Z}_{2}$ Nanocrystals}

$\mathrm{Er}^{3+}$ doped $\mathrm{ZrO}_{2}$ nanocrystals were synthesized by using the urea homogeneous precipitation method according to the reported protocols [4]. In a typical synthesis, metal salts with a stoichiometric mol ratio $(\mathrm{Zr} / \mathrm{Er}=100-\mathrm{x} / \mathrm{x}$, where $\mathrm{x}=$ 0.5, 1, 2 and $3 \mathrm{~mol} \%$ (a total of 0.001 mol for each sample)) were dissolved in $40 \mathrm{ml}$ of deionized DI water. Nanocrystals precipitates were prepared by heating the aqueous solution of corresponding metal salts with urea (40 $\mathrm{ml} \mathrm{DI} \mathrm{H}_{2} \mathrm{O}$ and $2 \mathrm{~g}$ urea each sample) in sealed glass battle at $140^{\circ} \mathrm{C}$ for $2 \mathrm{hrs}$. The resulting colloidal solutions were centrifuged at $4000 \mathrm{rpm}$ for $10 \mathrm{~min}$. The nanocrystals were washed 3 times with ethanol and DI water, dried and calcined in air at $800^{\circ} \mathrm{C}$ for $1 \mathrm{~h}$.

\subsection{Characterization}

The structure of the prepared powders was examined by XRD using a Bruker D8 Discover diffractometer (Bruker Optics Inc., MA, USA) with $\mathrm{Cu} \mathrm{K} \alpha$ radiation $(\lambda=0.15405$ $\mathrm{nm})$ and a $2 \theta$ scan range of 20 to $60^{\circ}$. The morphologies of the particles were characterized by FESEM (Hitachi S-4700, Hitachi, Ltd., Tokyo, Japan). The PL measurements were performed with a Hitachi F-7000 (Hitachi, Ltd., Tokyo, Japan) spectrophotometer equipped with a $150-\mathrm{W}$ xenon lamp as an excitation source. Additionally, to record the upconversion emission spectra of the $\mathrm{ZrO}_{2}: \mathrm{Er}^{3+}$, a commercially available $990 \mathrm{~nm}$ diode laser was used as the excitation source. Luminescence quantum yield (QY) was measured by a C9920-02 Hamamatsu absolute PL quantum yield measurement system (Hamamatsu Photonics K. K., Hamamatsu, Japan). All the measurements were performed at room temperature.

\section{Results and Discussion}

Figure 1 shows the XRD patterns taken for four samples with different concentrations. All samples exhibited identical diffraction patterns indicating a tetragonal phase (JCPDS no. 81-1544) [5]. No alien diffraction peaks of the other phases were detected, suggesting that the dopant ions had been well incorporated into the zirconia matrix to form a solid composite solution. All the diffraction peaks are sharp and have a strong intensity, showing that our samples have a very good crystallinity. This is very important for RE-doped phosphor materials because a good crystallinity means having fewer traps and stronger luminescence. However, since a crystalline symmetry depends on the concentration of the dopant, the overall peak intensity of the tetragonal phase $\mathrm{ZrO}_{2}$ indeed decreases with increasing of $\mathrm{Er}^{3+}$ concentration in nanocrystals.

The morphology of the synthesized phosphor particles after calcination at $800^{\circ} \mathrm{C}$ was examined by FESEM. Figure 2 shows the FESEM image of the $\mathrm{ZrO}_{2}: 1 \% \mathrm{Er}^{3+}$ nanocrystals. From the FESEM image, it is apparent that the $\mathrm{ZrO}_{2}: 1 \% \mathrm{Er}^{3+}$ nanocrystals consists of relatively small particles, about $80 \pm 20 \mathrm{~nm}$ in size. Doping with different $\mathrm{Er}^{3+}$ concentrations did not alter the morphology of the final product, and all samples had the similar morphology with a size distribution of $80 \pm 20 \mathrm{~nm}$ (not shown for other samples).

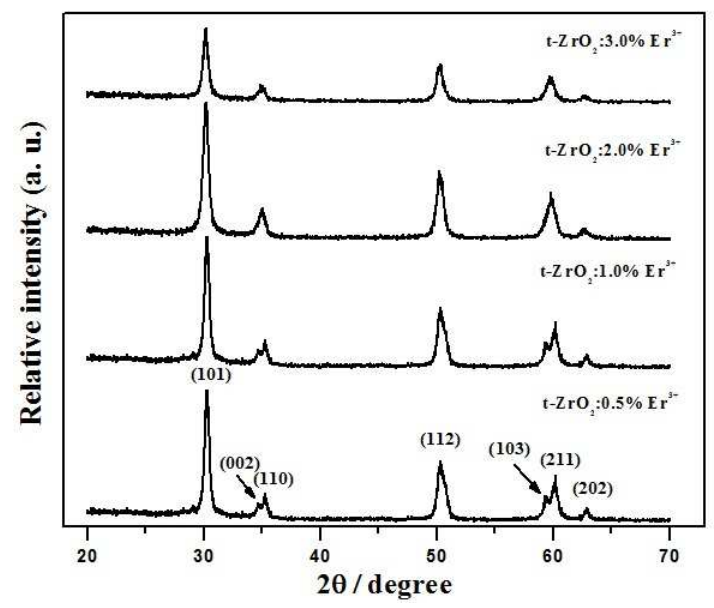

Figure 1. X-ray diffraction patterns of $\mathrm{ZrO}_{2}$ nanocrystals doped with different concentrations of $\mathrm{Er}^{3+}$.

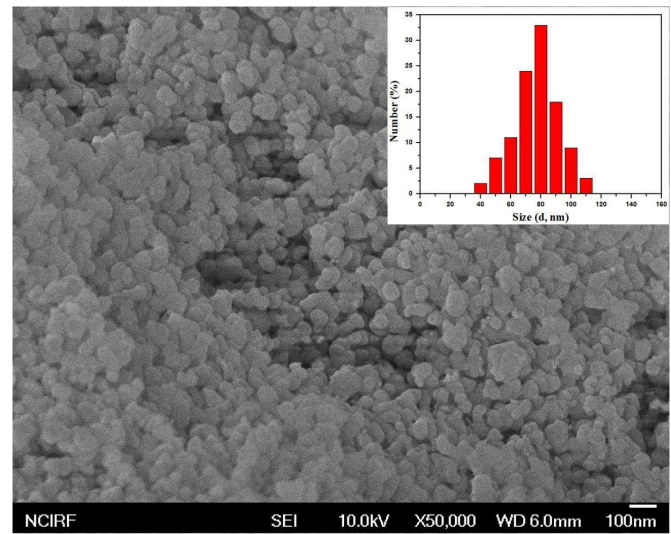

Figure 2. FESEM image and size distribution of $\mathrm{ZrO}_{2}: 1 \% \mathrm{Er}^{3+}$ nanocrystals.

The photoluminesce excitation and emission spectra of $\mathrm{ZrO}_{2}: 1 \% \mathrm{Er}^{3+}$ nanocrystals have been recorded under the near UV excitation at $378 \mathrm{~nm}$ as shown in Figure 3. The most intense excitation peak belongs to the hypersensitive transition to ${ }^{4} \mathrm{G}_{11 / 2}$. The normalized luminescence emission spectra of $\mathrm{ZrO}_{2}: 1 \% \mathrm{Er}^{3+}$ nanocrystals taken in the scan range from 475 to $700 \mathrm{~nm}$ under $378 \mathrm{~nm}$ (resonantly to the ${ }^{4} \mathrm{G}_{11 / 2}$ level) exhibits a strong green emission with some weak red emission. The sharp peaks in the green region were assigned to the ${ }^{2} \mathrm{P}_{3 / 2} \rightarrow{ }^{4} \mathrm{I}_{9 / 2},{ }^{2} \mathrm{H}_{11 / 2} ;{ }^{4} \mathrm{~S}_{3 / 2} \rightarrow{ }^{4} \mathrm{I}_{15 / 2}$ and ${ }^{2} \mathrm{H}_{9 / 2} \rightarrow$ ${ }^{4} \mathrm{I}_{13 / 2}$, whereas the peak in the red region correspond to the ${ }^{2} \mathrm{P}_{3 / 2} \rightarrow{ }^{4} \mathrm{~F}_{9 / 2}$ transition [6].

The luminescence emission of RE-doped phosphors for a frequency conversion is often affected by the dopant concentration. Therefore, it is very important to confirm the dependence of luminescence emission on the dopant concentration. To select the sample with high emission 
intensity, quantum yield QY of the samples was measured as a function of $\mathrm{Er}^{3+}$ concentration under a constant $378 \mathrm{~nm}$ UV excitation. Figure 4 shows that the QY of the samples increases with increasing dopant concentration up to 1 mol\% and then linearly decreased. The absolute QY values of $\mathrm{ZrO}_{2}: 0.5 \% \mathrm{Er}^{3+}, \mathrm{ZrO}_{2}: 1.0 \% \mathrm{Er}^{3+}, \mathrm{ZrO}_{2}: 2.0 \% \mathrm{Er}^{3+}$ and $\mathrm{ZrO}_{2}: 3 . \% \mathrm{Er}^{3+}$ were measured to be $1.6 \%, 2.3 \%, 1.1 \%$ and $0.4 \%$, respectively. This clearly suggests a dependence of the luminescence intensity on the concentration of dopant ions. The most reasonable explanation for this phenomenon is cross-relaxation mechanism within $\mathrm{Er}^{3+}$ ions. At low concentrations, dopant ions were rarely distributed in the host material. In other words, the number of emission centers is small. At higher dopant concentrations, the mean distance between dopant ions is much shorter; therefore ions can interact by an electric multipolar process leading to energy migration. The dipole-dipole quenching process is inversely proportional to the sixth power of ion-ion separation and thus to the square of the $\mathrm{Er}^{3+}$ concentration. And cross-relaxation process occurs followed by non-radiative decay of the two ions to the ground state. Thus, we can suppose that cross-relaxation mechanism occurs due to an interaction between the ions, energy migration, and nonradiative relaxation processes to the ground state [7].

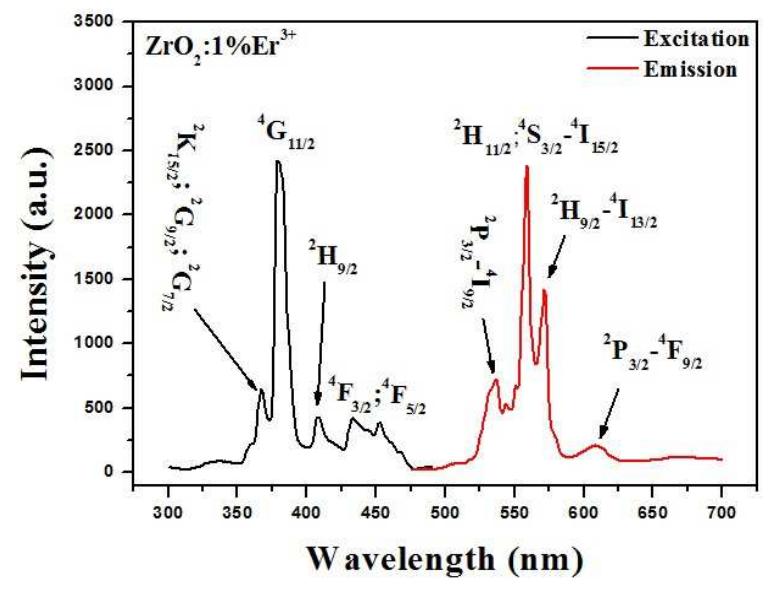

Figure 3. $\mathrm{PL}$ excitation and emission spectra of $\mathrm{ZrO}_{2}: 1 \% \mathrm{Er}^{3+}$ nanocrystals.

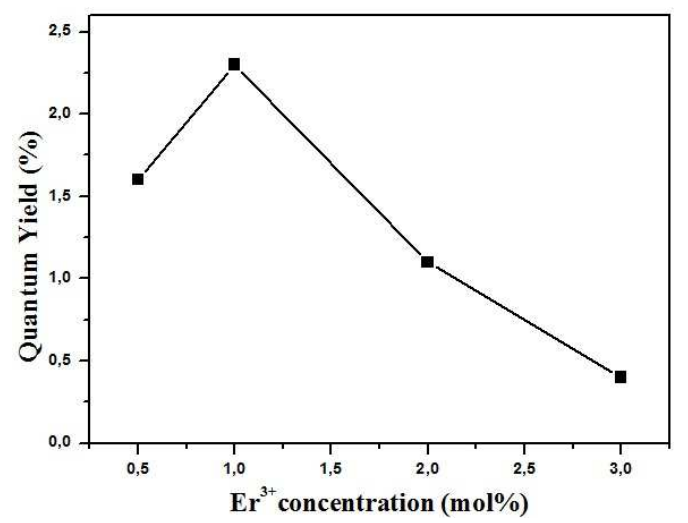

Figure 4. Measured $Q Y$ of the samples as a function of $\mathrm{Er}^{3+}$ concentration.
In addition, erbium is an excellent candidate for upconversion photoluminescence because the $4 f$ electronic levels can provide intermediate levels that are easily accessible with near IR radiation. Therefore, upconversion UC photoluminescence study was performed to demonstrate dual-modality of synthesized $\mathrm{ZrO}_{2}: \mathrm{Er}^{3+}$ nanocrystals. Figure 5 shows the normalized room-temperature $\mathrm{UC}$ spectra of $\mathrm{ZrO}_{2}: 1.0 \% \mathrm{Er}^{3+}$ nanocrystals (sample with optimum dopant concentration) under $990 \mathrm{~nm}$ near IR diode laser. The green emission between 540-585 mainly arises from ${ }^{2} \mathrm{H}_{11 / 2} ;{ }^{4} \mathrm{~S}_{3 / 2} \rightarrow{ }^{4} \mathrm{I}_{15 / 2}$ and ${ }^{2} \mathrm{H}_{9 / 2} \rightarrow{ }^{4} \mathrm{I}_{13 / 2}$ transitions and results from two-photon absorption processes [2]. Thus, the $\mathrm{ZrO}_{2}: 1.0 \% \mathrm{Er}^{3+}$ nanocrystals can be used as frequency convertors with both UV and near IR radiation.

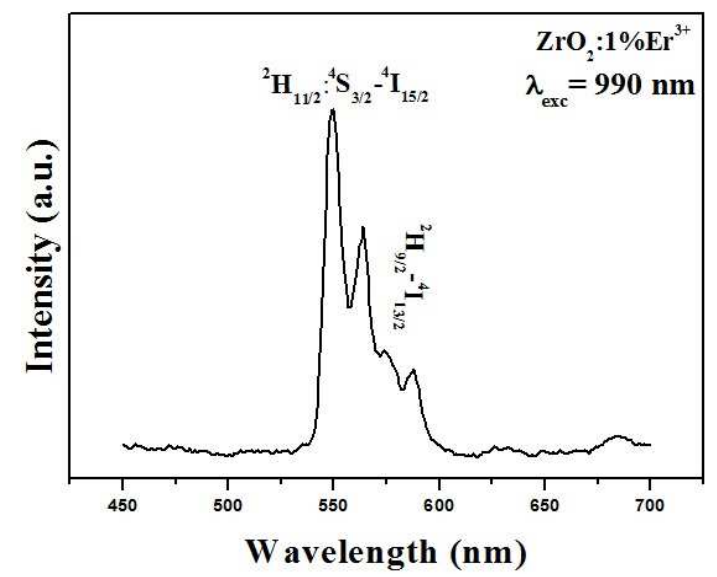

Figure 5. Upconversion emission spectra of $\mathrm{ZrO}_{2}: 1 \% \mathrm{Er}^{3+}$ nanocrystals.

\section{Conclusion}

Dual-mode luminescent $\mathrm{ZrO}_{2}: \mathrm{Er}^{3+}$ nanocrystals were successfully prepared via a facile and versatile urea homogeneous precipitation method. The optical properties of $\mathrm{ZrO}_{2}: \mathrm{Er}^{3+}$ nanocrystals have been systematically investigated. We demonstrated that the luminescence properties of synthesized $\mathrm{ZrO}_{2}: \mathrm{Er}^{3+}$ nanocrystals strongly depend on dopant concentration. Luminescence quenching was observed above $1 \mathrm{~mol} \%$ of $\mathrm{Er}^{3+}$ doped $\mathrm{ZrO}_{2}$ nanocrystals, which is considered to be the optimal dopant concentration. A strong green emission due to ${ }^{2} \mathrm{H}_{11 / 2} ;{ }^{4} \mathrm{~S}_{3 / 2} \rightarrow{ }^{4} \mathrm{I}_{15 / 2}$ transitions in $\mathrm{Er}^{3+}$ was observed upon both UV and near IR excitation. Dual-mode excitable $\mathrm{ZrO}_{2}: 1.0 \% \mathrm{Er}^{3+}$ nanocrystals are expected to find a wide range of applications, especially for solid state illumination and optoelectronic devices.

\section{Acknowledgements}

This work was financially supported by NRF research grant No 3348-20120033. We also gratefully acknowledge the project of Ehime University for promotion of international relations between SNU and EU for their financial supports. 


\section{References}

[1] Atabaev TS, Vu HHT, Piao Z, Hwang YH, Kim HK. Tailoring the luminescent properties of $\mathrm{Gd}_{2} \mathrm{O}_{3}: \mathrm{Tb}^{3+}$ phosphor particles by codoping with $\mathrm{Al}^{3+}$ ions. J Alloys Compd 541 (2012) 263-268

[2] Patra A, Friend CS, Kapoor R, Prasad PN. Upconversion in $\mathrm{Er}^{3+}: \mathrm{ZrO}_{2}$ Nanocrystals. J Phys Chem B 106 (2002) 1909-1912

[3] Liu Y, Zhou S, Tu D, Chen Z, Huang M, Zhu H, Ma E, Chen $\mathrm{X}$. Amine-functionalized lanthanide-doped zirconia nanoparticles: Optical spectroscopy, time-resolved fluorescence resonance energy transfer biodetection, and

targeted imaging. J Am Chem Soc 134 (2012) 15083-15090

[4] Atabaev TS, Kim HK, Hwang YH. Color-tunable properties of $\mathrm{Eu}^{3+}$ and $\mathrm{Dy}^{3+}$ codoped $\mathrm{Y}_{2} \mathrm{O}_{3}$ phosphor particles. Nanoscale Res Lett 7 (2012) 556

[5] Das S, Yang CY, Lu CH. Structural and optical properties of tunable warm-white light-emmiting $\mathrm{ZrO}_{2}: \mathrm{Dy}^{3+}-\mathrm{Eu}^{3+}$ nanocrystals. J Am Ceram Soc 96 (2013) 1602-1609

[6] Atabaev TS, Piao Z, Hwang YH, Kim HK, Hong NH. Bifunctional $\mathrm{Gd}_{2} \mathrm{O}_{3}: \mathrm{Er}^{3+}$ particles with enhanced visible upconversion luminescence. J Alloys Compd 572 (2013) 113-117

[7] Auzel F. Upconversion and anti-Stokes processes with $\mathrm{f}$ and $\mathrm{d}$ ions in solids. Chem Rev 104 (2004) 139-174 